\title{
Editorial
}

\section{JIDC approaches its fifth birthday}

\author{
Salvatore Rubino, Editor-in-Chief, JIDC
}

J Infect Dev Ctries 2012; 6(1):1-3.

(Received and Accepted 10 January 2012)

Copyright () 2012 Rubino. This is an open-access article distributed under the Creative Commons Attribution License, which permits unrestricted use, distribution, and reproduction in any medium, provided the original work is properly cited

The Journal of Infection in Developing Countries (JIDC) is approaching a significant milestone - the fifth anniversary of publishing our first issue in August 2007. Since posting that first issue online, JIDC has grown continuously and realized several important accomplishments. As a result of receiving an enthusiastic reception from the scientific community and the subsequent increase in submissions, we quickly moved from being a bimonthly to a monthly publication and were listed in several key databases including ISI Web of Knowledge (Science Citation Index Expanded), Index Copernicus, Chemical Abstracts, CABI Global Health, CAB Abstracts, Directory of Open Access Journals, CrossRef/DOI, HINARI (WHO), Ulrich's Periodicals Directory, Google Scholar, NLM Catalog, IMBIOMED, BOAI, WAME, COPE, DISUFSP(BR), GFMER(CH), IBT-CCG-UNAM(MX), WMA(ES), WB-LUMC(NL), LC(FR), BVUB(BEL), GU-L(US), L-YU(CA), HKUL(HK), RCL-UR (US), B-UNIFACS (BR), EBSCO . Then in August 2009, we were thrilled to be accepted into the distinguished PubMed database.

Our next goal is to receive an impact factor, and in a few months we will receive our impact factor evaluation from Thompson Reuters. Looking at Google Scholar, we have about 1,150 citations with a constant monthly increase, so we are anticipating good news. We can only go up from here! We are eternally grateful to our readers who inspire us to present the important research from developing countries.

We are very proud of our publishing record. In 2011 JIDC published a total of 155 papers including reviews, regional reviews, outbreak reports, original papers, case reports, technical notes, and letters written by authors from many different developing countries. We greatly appreciate the contributions of our authors (733) who hail from 358 institutions in 60 countries, without whom our accomplishments would not have been possible.

JIDC is based on the hard work of volunteers who believe in the JIDC project and give their time to achieve JIDC's goal of reducing the scientific gap experienced by developing countries.

This is the right time to thank the editorial team including our senior editors, editors, associate editors and members of editorial board. We greatly appreciate the excellent work of our core team, including chief technical editor and production coordinator Nikki Kelvin, webmaster and production coordinator Marco Scano, page setter Joey Kelvin, creative director Jeff Coombs, and administrative assistant Giustina Casu.

Particular thanks go to our scientific editors Nadia Ahmod, Eve Boakes, Mark Cameron, Cheryl Cameron, Antigoni Chaidaroglou, Marie Anne Chattaway, Daniela Chessa, Amber Farooqui, Katy Harker, Jorg Heukelbach, Richard C. Huard, Adnan Khan, Patrick J. Kelly, Gemma C Langridge, Asghar Nazeer, Alicia San José, Helena Seth-Smith, Chengming Wang, Aysegul Karahasan Yagci, and all the editors, reviewers, and board members who answered our call for science editors in October.

We are very grateful to our dedicated technical editors, Dominique China, Mark David Dickson, Emily Hiltz, Lisa M. Lambert, and Eva Molak.

Special thanks must be given as well to our senior editor, Aysegul Karahasan Yagci, who prepared our current special issue on tuberculosis.

Everyone on our editorial team is committed to our mentoring system, which is at the heart of JIDC, starting at the pre-submission level and carried through the entire publishing process.

We are also very proud of our successful blog, which is new to JIDC this past year. Headed masterfully and with a great dedication by Alyson Kelvin, it is a place where professional experiences 
and scientific ideas can be exchanged. JIDC also has a Facebook page, where you can get updates on our editorial meetings and view our interesting gallery of JIDC friends, and we are followed on Twitter. We also publish a monthly newsletter that is sent to subscribers.

The financial burden of maintaining JIDC is shouldered by JIDC volunteers and grants and donations from foundations and organizations such as Fondazione Banco di Sardegna, Sardegna Ricerche, the University of Sassari, Shantou University Medical College, the Li Ka Shing Foundation, and the University Health Network in Toronto, Canada. Our heartfelt gratitude goes out to these people and organizations.

Finally, we are very grateful to all the reviewers who contribute their precious time helping with suggestions for revisions and ensuring that the journal maintains high professional standards. While our reviewers are from 65 countries from all over the world, most of them are from developing countries. We are fortunate to have reviewers from the following countries: Argentina, Australia, Austria, Bangladesh, Belgium, Brazil, Burkina Faso, Canada, Chile, China, Costa Rica, Croatia, Egypt, Ethiopia, France, Gabon, Gambia, Georgia, Germany, Greece, Hong Kong, India, Indonesia, Iran, Iraq, Italy, Kenya, Kuwait, Lebanon, Libya, Madagascar, Malaysia, Mexico, Morocco, Nepal, Netherlands, New Caledonia, New Zealand, Nigeria, Pakistan, Panama, Paraguay, Perù, Poland, Portugal, Russia, Saint Kitts and Nevis, Saudi Arabia, Syria, South Africa, Spain, Sudan, Taiwan, Tanzania, Thailand, Tunisia, Turkey, Uganda, United Kingdom, United Arab Emirates, Uruguay, United States, Venezuela, Vietnam, Zimbabwe.

We would like to acknowledge and thank the following 257 experts who reviewed the papers submitted for consideration in our 2011volume:

Lorena Abadia-Patino (Venezuela), Ibrahim AbdelMessih (Italy), Yusuf Abdu Misau (Nigeria), Hikmate Abriouel (Spain), Ishag Adam (Sudan), Mohammad Aghasadeghi (Iran), Kabiru Akinyemi (Nigeria), Onoja Akpa (Nigeria), Bolajoko Aina (Nigeria), Raquel Almansa (Spain), Sitara Ajjampur (India), Carlos Alencar (Brazil), Abdulghani Alsamarai (Iraq), Patricia Agaba (Nigeria), Saurabh Agarwal (India), Deepak Aggarwal (United States), Jaffar Al-Tawfiq (Saudi Arabia), Carlos AmabileCuevas (Mexico), Malaka Amer (Saudi Arabia), Ben Amos (Tanzania), Vicente Antonello (Brazil),
Maria Appleman (United States), Liana Ariza (Brazil), George Araj (Lebanon), Antonio Arbo (Paraguay), Carmen Ardanuy (Spain), Elisabete Arrese (Spain), Ifedayo Adetifa (Gambia), Samar AlNahhas (Syria), Ibrahim Al-Traif (Saudi Arabia), Jazzmin Arrivillaga (Venezuela), Myo Aung (Thailand), Reham A. Aziz (Egypt), Sina Aziz (Pakistan) Ahmed Al-Naaimi (Iran), Clement Azodo (Nigeria), Parisa Badiee (Iran), Ahmet Balik (Turkey), Frederick Baliraine (United States), Kristin Banek (Uganda), Bita Bakhshi (Iran), Stephen Baker (Vietnam), Mustafa Bakir (Turkey), Fukai Bao (China), Oussama Baraket (Tunisia), Ladaporn Bodhidatta (Thailand), Andreas Baumler (United States), Wafaa Bakr (Egypt), Matteo Biasotto (Italy), Norma Binsztein (Argentina), Joseph Bliss (United States), Eve Boakes (United Kingdom), Steven Bosinger (United States), Giuseppe Botta (Italy), Zulkif Bozgeyik (Turkey), Maria Braoudaki (Greece), John Bremner (Australia), Rama Bhunia (India), Mathilde Boon (Netherlands), Franco Buonaguro (Italy), Cyrille Bisseye (Burkina Faso), Jorge Cabral (Portugal), Adrian Canizalez-Roman (Mexico), Jean-Philippe Chippaux (France), Andrea Carai (Italy), Nishat Chowdhury (Bangladesh), Lance Christiansen (United States), Phillip Collier (United Kingdom), Mauro Colombo (Italy), Daniela Ceccarelli (Italy), Solmaz Celebi (Turkey), Changfa (Clark) Cui (United States), Marie Chattaway (United Kingdom), Giancarlo Ceccarelli (Italy), Ricardo Correa (Panama), Carlos Canelo-Aybar (Peru), Nikoloz Chkhartishvili (Georgia), Rajesh Chudasama (India), Celso Cunha (Portugal), Nihar Dash (United Arab Emirates), Terezinha da Silva Leitao (Brazil), Monica da Silva-Nunes (Brazil), Barun Kumar De (United States), Monorama Deb (India), Debmita Debdas (India), Bouke De Jong (Belgium), Mireya de la Garza (Mexico), Giovanni Delogu (Italy), Jacobus de Waard (Venezuela), Elisa Deriu (United States), İlker Devrim (Turkey), Antonina Dolei (Italy), Gerusa Dreyer (Brasil), Maria Drogari-Apiranthitou (Greece), Milena Dropa (Brazil), Renu Dutta (India), Shanta Dutta (India), Mohamad Elsweify (Saudi Arabia), Atef El-Gendy (Egypt), Sabry El-Naggar (Egypt), Şevval Eren (Turkey), Abdul-Naser Elzouki (Libya), Teresa Estrada-Garcia (Mexico), Mônica Facanha (Brazil), Heriberto Fernandez (Chile), Joseli Ferreira (Brazil), Parissa Farnia (Iran), Mel Figtree (Australia), Joseph Forbi (Nigeria), Lucia Galli (Italy), R. Glenn Gaston (United States), Gerardo González-Rocha (Chile), P. Grabarczyk (Poland), Javier Garaizar (Spain), Marina Giuliano (Italy), 
Deniz Gur Akman (Turkey), Tayyaba Ijaz (Pakistan), Noyal Joseph (India), Yadav Prasad Joshi (Nepal), Wafa Habbal (Sirya), Xiang Han (United Stetes), Azian Harun (Malaysia), Abdul Haque (Pakistan), Zahra Hasan (Pakistan), Mary Hoelscher (United States), Richard C. Huard (United States), Entesar Husain (Kuwait), Murat Kapan (Turkey), Ismail Kapicioglu (Turkey), Oguz Karabay (Turkey), Salim Abdool Karim (South Africa), Supaletchimi Katherason (Malaysia), Rumeyza Kazancioğlun (Turkey), Adnan Khan (Pakistan), Karen Keddy (South Africa), Abdullah Kilic (Turkey), Paul Klatser (Netherlands), Diamantis Kofteridis (Greece), Michael Koster (United States), Anil Kumar (India), J. Labbe (New Caledonia), Lincoln Lau (Hong Kong), Sisinnio Leori (Italy), Benedikt Ley (Austria), Ming-Li Liou (Taiwan), Jean Limongi (Brazil), Amanda Loftis (Saint Kitts and Nevis), Zhigang Liu (United States), Chun-Yi Lu (Taiwain), Eric Macy (United States), Emin Maden (Turkey), Evelyn Madoroba (South Africa), Stanley Maloy (United States), Daniel Marcano (Venezuela), Margarita Martinez-Medina (Spain), Humphrey Mazigo (Tanzania), Jacobe Mathew (India), Mark Melzer (United Kingdom), Juliane Meng-Hentschel (Germany), Vitaliano Muzii (Italy), Lucky Moehario (Indonesia), Denise Patricia Mawili-Mboumba (Gabon), Leigh Miller (Thailand), Santosh Mondal (India), David Murdoch (New Zealand), Reggie Mutetwa (Zimbabwe), Nutan Mytle (USA), Joseph Mwanzia Nguta (Kenya), Gholamreza Nikbakht Brujeni (Iran), Vishal Nigam (India), Carla Novais (Portugal), Samuel Ola (Nigeria), Obeid Obeid (Saudi Arabia), A. B. Olokoba (Nigeria), Bianca Paglietti (Italy), Tibor Pal (United Arab Emirates), Dimple Panwar (India), Georgios Pappas (Greece), Violeta Pardío (Mexico), Luis Pianciola (Argentina), Ariel Pereda (Argentina), Alicia Perez (France), Celeste Pérez (Argentina), Cristian Perez (Costa Rica), Mrudula Phadke (India), Guillermo Pimentel
(United States), Aggeliki Poulou (Greece), Farhad Pourfarzi (Iran), Audino Podda (Italy), Mayoor Prabhu (India), Pradyot Prakash (India), Zhikang Qian (United States), Manuela Raffatellu (United States), Md Siddiqur Rahman (Bangladesh), Paola Rappelli (Italy), Ayalu Reda (Ethiopia), Ana Carolina Ritter (Brazil), Octavio Rivero (Spain), Alesia Romanovkaya (Russia),Jane Rose (Saint Kitts and Nevis), Joaquim Ruiz (Spain), Suleiman Saidi (Kenya), Eisa Salehi (Iran), Sandeep Saluja (India), Vittorio Sambri (Italy), Amy Sapkota (United States), Sanda Sardelic (Croatia), Shiv Sarin (India), Murat Sayan (Turkey), Dianella Savoia (Italy), Karin Schwaiger (Germany), M Elena Seoane (Spain), Burcin Sener (Turkey), Asa Sjoling (Sweden), Abubaker Sidahmed (Canada), Nidhi Singla (India), Herman Sprenger (Netherlands), Ajanta Sharma (India), Seyed Davar Siadat (Iran), Addmore Shonhai (South Africa), Kirill Sharshov (Russia), VN Sehgal (India), Michael Seckeler (United States), Pierre Smeesters (Belgium), Malini Shariff (India), Wei Sun (United States), Olusegun Soge (United States), Bahman Tabaraie (Iran), Irene Tami (United States), Reda Tagajdid (Morocco), Narhari Timilshina (Canada), Mohammed Timinouni (Morocco), Eduardo Tondo (Brazil), Hasan Ucmak (Turkey), Uade Ugbomoiko (Nigeria), Kingsley Ukwaja (Nigeria), Maria Leide Wand-del-Rey Oliveira (Brazil ), Tjip van der Werf (Netherlands), A Veloo (Netherlands), Tommie Victor (South Africa), Roberto Vidal (Chile), Ha Vinh (Vietnam), Jugsharan Virdi (India), Simonetta Viviani (France), Georgia Vrioni (Greece), Richard Vincent (Madagascar), Francois-Xavier Weill (France), Hannah Wexlecheck (United States), Heather Williamson (United States), Paul-Louis Woerther (France), Asnakew Yeshiwondim (United States), Raffaele Zarrilli (Italy), Dominik Zenner (United Kingdom), Roberto Zenteno (Mexico), Abdulaziz Zorgani (Libya), Pablo Zunino (Uruguay). 